\title{
A Fast Algorithm to Solve the Bitsadze Equation in the Unit Disk
}

\author{
Daoud Mashat, Manal Alotibi \\ Department of Mathematics, Faculty of Science, King Abdulaziz University, Jeddah, Saudi Arabia \\ E-mail:dmashat@kau.edu.sa,dmashat63@gmail.com \\ Received October 19, 2010; revised November 18, 2010; accepted November 24, 2010
}

\begin{abstract}
An algorithm is provided for the fast and accurate computation of the solution of the Bitsadze equation in the complex plane in the interior of the unit disk. The algorithm is based on the representation of the solution in terms of a double integral as it shown by Begehr [1,2], some recursive relations in Fourier space, and Fast Fourier Transforms. The numerical evaluation of integrals at $N^{2}$ points on a polar coordinate grid by straightforward summation for the double integral would require $O\left(N^{2}\right)$ floating point operation per point. Evaluation of such integrals has been optimized in this paper giving an asymptotic operation count of $O(\operatorname{In} N)$ per point on the average. In actual implementation, the algorithm has even better computational complexity, approximately of the order of $O(1)$ per point. The algorithm has the added advantage of working in place, meaning that no additional memory storage is required beyond that of the initial data. This paper is a result of application of many of the original ideas described in Daripa [3].
\end{abstract}

Keywords: Singular Integrals, Fast Algorithm, Bitsadze Equation

\section{Introduction}

The solutions of many elliptic partial differential equations represent in terms of singular integrals in the complex plane in the interior of the unit disk, as the nonhomogeneous Cauchy-Riemann equations, the Beltrami equation, the Poisson equation, etc. Then solving these equations requires computing the values of the singular integrals. There are two main difficulties in the straightforward computation of these integrals using quadrature rules. Firstly, straightforward computation of each of these integrals requires an operation count of the order $O\left(N^{2}\right)$ per point. This gives a net operation count of $O\left(N^{4}\right)$ for $N^{2}$ grid points which is computationally very intensive for large $N$. Secondly, this method also gives poor accuracy due to the presence of the singularities in the integrand. Daripa and Co-workers ([3-9]) presented fast algorithms to solve the singular integrals that arise in such solutions. By these algorithms evaluation of singular integrals has been optimized, giving an asymptotic operation count of $O\left(N^{2} \ln \ln N\right)$ for $N^{2}$ points. Moreover, these algorithms have the added advantages of working in place, meaning that no additional memory storage is required beyond that of the initial data.
In this paper we follow his method to present an algorithm to solve an elliptic partial differential equation called the Bitsadze equation which define in the unit disk $B(0 ; 1)=\{z:|z|<1\}$ in complex plane $\mathbb{C}$ for any complex valued function $w$ of complex variables $z, \bar{z} \in B$ by

$$
\frac{\partial^{2}}{\partial \bar{z}^{2}} w \equiv \frac{1}{4}\left(\frac{\partial}{\partial x}+i \frac{\partial}{\partial y}\right)^{2} w=f ; z=x+i y, x, y \in \mathbb{C}
$$

Where $f$ is a complex valued function on $B$. [10]

The Bitsadze equation arises in many areas including structural mechanics, electrostatics, magneto statics, power electromagnetic, conductive media, heat transfer and diffusion ([11-13]). In [1,2], Begehr introduced some boundary value problems for Bitsadze equation under some solvability conditions and presented their solutions as results for applying the Dirichlet and Neumann boundary value problems all those solutions have singular integrals in their context. For that, we will consider one of these problems to apply our numerical method for evaluating the singular integrals.

Problem (1.1):

$w_{\overline{z Z}}=f$ in $B, w=\gamma_{0}, z w_{z \bar{z}}=\gamma_{1}$ on $\partial B, w_{\bar{z}}(0)=c$,

Where $f \in L_{1}(B ; \mathbb{C}), \gamma_{0}, \gamma_{1} \in C(\partial B ; \mathbb{C})$ and $c \in \mathbb{C}$. 
Its unique solution is given by Equation (1.2) (Below)

Let us rewrite it in the form

$$
w(z)=c \bar{z}+u(z)+v(z)+G f(z),
$$

Where

$$
\begin{gathered}
u(z)=\frac{1}{2 \pi i} \int_{|\zeta|=1} \gamma_{0}(\zeta) \frac{d \zeta}{\zeta-z}, \\
v(z)=\frac{1}{2 \pi i} \int_{|\zeta|=1} \gamma_{1}(\zeta) \frac{1-|z|^{2}}{z} \log (1-z \bar{\zeta}) \frac{d \zeta}{\zeta},
\end{gathered}
$$

and

$$
G f(z)=\frac{1}{\pi} \iint_{|\zeta|<1} f(\zeta) \frac{|\zeta|^{2}-|z|^{2}}{\zeta(\zeta-z)} d \xi d \eta
$$

Our method is basically a recursive routine in Fourier space that divides the interior of the unit disk into a collection of annular regions and expands the integral in Fourier series in angular direction with radius dependent Fourier coefficients. A set of exact recursive relations are derived which are used to produce the Fourier coefficients of singular. These recursive relations involve appropriate scaling of one-dimensional integrals in annular regions. The integrals in (1.4) at all grid points are then easily obtained from the Fourier coefficients by the FFT.

The rest of the paper is structured as follows. Section 2 presents the solution of the linear integrals $u$ and $v$. Section 3 develops the mathematical foundation of the efficient algorithm to evaluate (1.4) within the unit disk. The fast algorithms for solving problem (1.1) is discussed in Section 4 . We carry out numerical results with this method in section 5. Finally, we summarize and conclude in Section 6.

\section{Evaluating the Integrals $u$ and $v$}

1)

$$
u(z)=\frac{1}{2 \pi i} \int_{|\zeta|=1} \gamma_{0}(\zeta) \frac{d \zeta}{\zeta-z}
$$

Let $\zeta=\rho e^{i \tau}$ and $z=r e^{i \alpha}$, where $\rho, r \neq 0$ then

$$
u\left(r e^{i \alpha}\right)=\frac{1}{2 \pi i} \int_{0}^{2 \pi} \gamma_{0}\left(\rho e^{i \tau}\right) \frac{i \rho e^{i \tau} d \tau}{\rho e^{i \tau}-r e^{i \alpha}},
$$

Since the integral is on the unit disk then $\rho=1$,

$$
\begin{aligned}
u(r, \alpha) & =\frac{1}{2 \pi} \int_{0}^{2 \pi} \frac{\gamma_{0}\left(e^{i \tau}\right)}{1-r e^{i(\alpha-\tau)}} d \tau \\
& =\sum_{n=0}^{\infty} \frac{1}{2 \pi} \int_{0}^{2 \pi} \gamma_{0}\left(e^{i \tau}\right) r^{n} e^{i n(\alpha-\tau)} d \tau
\end{aligned}
$$

Let $\gamma_{0}\left(e^{i \tau}\right)=\sum_{n=-\infty}^{\infty} a_{n} e^{i n \tau}$,

$$
\begin{aligned}
u(r, \alpha) & =\sum_{n=0}^{\infty} \frac{1}{2 \pi} \int_{0}^{2 \pi}\left[\sum_{n=-\infty}^{\infty}\left(a_{n} r^{n} e^{i n \alpha}\right) e^{i n \tau}\right] e^{-i n \tau} d \tau \\
& =\sum_{n=0}^{\infty} a_{n} r^{n} e^{i n \alpha},
\end{aligned}
$$

Then we can write

$$
u(r, \alpha)=\sum_{n=-\infty}^{\infty} u_{n} e^{i n \alpha}
$$

Where

$$
u_{n}= \begin{cases}a_{n} r^{n} & \text { if } n \geq 0 \\ 0 & \text { if } n<0\end{cases}
$$

2) $v(z)=\frac{1}{2 \pi i} \int_{|\zeta|=1} \gamma_{1}(\zeta) \frac{1-|z|^{2}}{z} \log (1-z \bar{\zeta}) \frac{d \zeta}{\zeta}$,

$$
\begin{aligned}
v(r, \alpha) & =\frac{1}{2 \pi i} \int_{0}^{2 \pi} \gamma_{1}\left(\rho e^{i \tau}\right) \frac{1-r^{2}}{r e^{i \alpha}} \log \left(1-r e^{i \alpha} \rho e^{-i \tau}\right) \frac{i \rho e^{i \tau} d \tau}{\rho e^{i \tau}}, \\
& =\frac{1-r^{2}}{2 \pi r e^{i \alpha}} \int_{0}^{2 \pi} \gamma_{1}\left(e^{i \tau}\right) \log \left(1-r e^{i(\alpha-\tau)}\right) d \tau, \\
& =\sum_{n=1}^{\infty} \frac{r^{2}-1}{2 \pi r e^{i \alpha}} \int_{0}^{2 \pi} \gamma_{1}\left(e^{i \tau}\right) \frac{r^{n}}{n} e^{i n(\alpha-\tau)} d \tau .
\end{aligned}
$$

Let $\gamma_{1}\left(e^{i \tau}\right)=\sum_{n=-\infty}^{\infty} b_{n} e^{i n \tau}$,

$$
\begin{aligned}
v(r, \alpha) & =\sum_{n=1}^{\infty} \frac{r^{2}-1}{r e^{i \alpha}} \frac{1}{2 \pi} \int_{0}^{2 \pi}\left[\sum_{n=-\infty}^{\infty}\left(\frac{b_{n} r^{n}}{n} e^{i n \alpha}\right) e^{i n \tau}\right] e^{-i n \tau} d \tau, \\
& =\sum_{n=1}^{\infty} \frac{b_{n}}{n}\left(r^{n+1}-r^{n-1}\right) e^{i \alpha(n-1)} .
\end{aligned}
$$

put $n=m+1$

$$
v(r, \alpha)=\sum_{m=0}^{\infty} \frac{b_{m+1}}{m+1}\left(r^{m+2}-r^{m}\right) e^{i m \alpha} .
$$

Then we can write

$$
v(r, \alpha)=\sum_{m=-\infty}^{\infty} v_{m} e^{i m \alpha},
$$

Where

$$
v_{m}= \begin{cases}\frac{b_{m+1}}{m+1}\left(r^{m+2}-r^{m}\right) & \text { if } m \geq 0 \\ 0 & \text { if } m<0\end{cases}
$$

$$
w(z)=c \bar{z}+\frac{1}{2 \pi i} \int_{|\zeta|=1} \gamma_{0}(\zeta) \frac{d \zeta}{\zeta-z}+\frac{1}{2 \pi i} \int_{|\zeta|=1} \gamma_{1}(\zeta) \frac{1-|z|^{2}}{z} \log (1-z \bar{\zeta}) \frac{d \zeta}{\zeta}+\frac{1}{\pi} \iint_{|\zeta|<1} f(\zeta) \frac{|\zeta|^{2}-|z|^{2}}{\zeta(\zeta-z)} d \xi d \eta \text {. }
$$




\section{Mathematical Foundation of the Algorithms}

In this section, we develop the theory needed to construct an efficient algorithm for evaluation the singular integrals (1.4). The following theorem is crucial for later development of the algorithm.

Theorem 3.1. The value of the integral (1.4) with $z=r e^{i \alpha}, \quad r \neq 0$ can be written as

$$
G f(z)=\sum_{n=-\infty}^{\infty} g_{n}(r) e^{i n \alpha}
$$

Where

$$
g_{n}(r)=\frac{1}{\pi} \begin{cases}\iint_{B(0 ; 1)-B(0 ; r)} f(\zeta) \frac{|\zeta|^{2} r^{n}-r^{n+2}}{\zeta^{n+2}} d \xi d \eta ; & n \geq 0 \\ \iint_{B(0 ; r)} f(\zeta) \frac{r^{n}\left(r^{2}-|\zeta|^{2}\right)}{\zeta^{n+2}} d \xi d \eta ; & n \leq 1\end{cases}
$$

Proof. If we denote

$$
Q(r, \zeta)=\frac{1}{2 \pi} \int_{0}^{2 \pi} \frac{|\zeta|^{2}-|z|^{2}}{\zeta(\zeta-z)} e^{-i n \alpha} d \alpha
$$

Then it follows from (1.4) and (3.1) that

$$
g_{n}(r)=\frac{1}{\pi} \iint_{B(0 ; 1)} f(\zeta) Q(r, \zeta) d \xi d \eta .
$$

The integral in (3.3) can be evaluated by first expanding $\frac{|\zeta|^{2}-|z|^{2}}{\zeta(\zeta-z)}$ in power of $\frac{\zeta}{z}$ and $\frac{z}{\zeta}$ to get

$$
\frac{|\zeta|^{2}-|z|^{2}}{\zeta(\zeta-z)}= \begin{cases}\sum_{n=0}^{\infty} \frac{r^{n}\left(|\zeta|^{2}-r^{2}\right)}{\zeta^{n+2}} e^{i n \alpha} ; & r<|\zeta|, \\ \sum_{n \leq 1} \frac{r^{n}\left(r^{2}-|\zeta|^{2}\right)}{\zeta^{n+2}} e^{i n \alpha} ; & r>|\zeta| .\end{cases}
$$

By comparing this result with the Fourier series coefficients of $\frac{|\zeta|^{2}-|z|^{2}}{\zeta(\zeta-z)}$, we get

$$
Q(r, \zeta)= \begin{cases}0 ; & n \geq 0, r>|\zeta|, \\ \frac{|\zeta|^{2} r^{n}-r^{n+2}}{\zeta^{n+2}} ; & n \geq 0, r<|\zeta|, \\ \frac{r^{n+2}-|\zeta|^{2} r^{n}}{\zeta^{n+2}} ; & n \leq-1, r>|\zeta|, \\ 0 ; & n \leq-1, r<|\zeta| .\end{cases}
$$

Substitution of (3.5) into (3.4) yields the desired result.

Corollary 3.1. Suppose that $\zeta=\rho e^{i \theta}$ and $f(\zeta)$ has Fourier coefficient $f_{n}(\rho)$; then the coefficients $g_{n}(r)$ in Equation (3.2) can be written as:

$$
g_{n}(r)=2 \begin{cases}\int_{r}^{1} \frac{\rho^{2} r^{n}-r^{n+2}}{\rho^{n+1}} f_{n+2}(\rho) d \rho ; & n \geq 0, \\ \int_{0}^{r} \frac{r^{n+2}-\rho^{2} r^{n}}{\rho^{n+1}} f_{n+2}(\rho) d \rho ; & n \leq-1 .\end{cases}
$$

Corollary 3.2. It follows directly from Equation (3.6) that

$$
g_{n}(1)=0 \text { for } n \geq 0, g_{n}(0)=0 \text { for } n \leq-1 \text {. }
$$

Corollary 3.3. Let $r_{i}<r_{j}$. Define

$$
C_{i, j}^{n}= \begin{cases}\int_{r_{i}}^{r_{j}} \frac{f_{n+2}(\rho)}{\rho^{n-1}} d \rho ; & n \geq 0, \\ \int_{r_{i}}^{r_{j}} \frac{f_{n+2}(\rho)}{\rho^{n+1}} d \rho ; & n \leq-1 .\end{cases}
$$

and

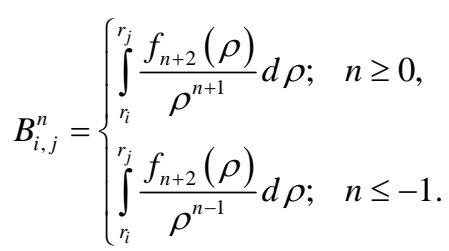

After some algebraic manipulation it follows from Equations (3.7) and (3.8) that

$$
g_{n}\left(r_{i}\right)=C_{n}\left(r_{i}\right)-B_{n}\left(r_{i}\right)
$$

Where

$$
\begin{gathered}
C_{n}\left(r_{i}\right)=2 r_{i}^{n} C_{i, j}^{n}+\left(\frac{r_{i}}{r_{j}}\right)^{n} C_{n}\left(r_{j}\right) ; n \geq 0, \\
C_{n}\left(r_{j}\right)=\left(\frac{r_{i}}{r_{j}}\right)^{-n-2} C_{n}\left(r_{i}\right)+2 r_{j}^{n+2} C_{i, j}^{n} ; n \leq-1, \\
B_{n}\left(r_{i}\right)=2 r_{i}^{n+2} B_{i, j}^{n}+\left(\frac{r_{i}}{r_{j}}\right)^{n+2} B_{n}\left(r_{j}\right) ; n \geq 0,
\end{gathered}
$$

and

$$
B_{n}\left(r_{j}\right)=\left(\frac{r_{i}}{r_{j}}\right)^{-n} B_{n}\left(r_{i}\right)+2 r_{j}^{n} B_{i, j}^{n} ; n \leq-1 .
$$

Corollary 3.4. Let $0=r_{0}<r_{1}<r_{2}<\cdots<r_{M}=1$. It follows from recursive applications of (3.10)-(3.13) and from using Corollary 3.2 that

$$
g_{n}\left(r_{l}\right)=\left\{\begin{array}{l}
2 \sum_{i=l}^{M-1}\left(r_{l}^{n} C_{i, i+1}^{n}-r_{l}^{n+2} B_{i, i+1}^{n}\right) ; n \geq 0, \\
2 \sum_{i=1}^{l}\left(r_{l}^{n+2} C_{i-1, i}^{n}-r_{l}^{n} B_{i-1, i}^{n}\right) ; n \leq-1 .
\end{array}\right.
$$


for $l=1,2,3, \cdots, M-1$.

Corollary 3.5. It follows directly from Equations (3.6) and (3.9) that

$$
\begin{gathered}
C_{n}(1)=B_{n}(1)=0 ; n \geq 0, \\
C_{n}(0)=B_{n}(0)=0 ; n \leq-1 .
\end{gathered}
$$

\section{The Fast Algorithm}

We construct the fast algorithm based on the theory of section 3. The unit disk is discredited using $M \times N$ lattice points with $M$ equidistant points in the radial direction and $N$ equidistant points in the circular direction. The following is a formal description of the fast algorithm useful for programming purposes.

Algorithm 4.1. (The fast algorithm for evaluating the singular integral (1.4))

Input: $M, N$ and $f\left(r_{l} e^{2 \pi i k / N}\right), l \in[1, M-1], k \in[1, N]$

Output: $G f\left(r_{l} e^{2 \pi i k / N}\right), l \in[1, M-1], k \in[1, N]$.

Step 1. Set $K=N / 8, r_{0}=0$ and $r_{M}=1$.

Step 2. Compute the Fourier coefficients $f_{n}\left(r_{l}\right)$, $\forall l \in[0, M-1]$ and $n \in[-K, K]$ from known values of $f\left(r_{l} e^{2 \pi i k / N}\right), k=1,2, \cdots, N$ using the FFT.

Step 3. Compute $C_{i, i+1}^{n} \forall i \in[1, M-1]$ and $n \in[-K-2,-1] \cup[0, K-2]$ using Equation (3.7).

Step 4. Compute $B_{i, i+1}^{n} \forall i \in[1, M-1]$ and $n \in[-K-2,-1] \cup[0, K-2]$ using Equation (3.8).

Step 5. Compute $g_{n}\left(r_{l}\right), n \in[-K-2, K-2]$, $l \in[1, M-1]$ using relations (3.14).

$$
\begin{aligned}
& \text { set } C_{n}\left(r_{M}\right)=0, B_{n}\left(r_{M}\right)=0, \forall n \in[0, K-2] \\
& \text { do } n=0,1, \cdots, K-2 \\
& \text { do } l=M-1, \cdots, 1 \\
& C_{n}\left(r_{l}\right)=2 r_{l}^{n} C_{l, l+1}^{n}+\left(\frac{r_{l}}{r_{l+1}}\right)^{n} C_{n}\left(r_{l+1}\right), \\
& B_{n}\left(r_{l}\right)=2 r_{l}^{n+2} B_{l, l+1}^{n}+\left(\frac{r_{l}}{r_{l+1}}\right)^{n+2} B_{n}\left(r_{l+1}\right), \\
& g_{n}\left(r_{l}\right)=C_{n}\left(r_{l}\right)-B_{n}\left(r_{l}\right) . \\
& \text { enddo } \\
& \text { enddo } \\
& \text { set } C_{n}\left(r_{0}\right)=0, B_{n}\left(r_{0}\right)=0, \forall n \in[-K-2,-1] \\
& C_{n}\left(r_{1}\right)=2 r_{1}^{n+2} C_{0,1}^{n}, \\
& B_{n}\left(r_{1}\right)=2 r_{1}^{n} B_{0,1}^{n}, \\
& g_{n}\left(r_{1}\right)=C_{n}\left(r_{1}\right)-B_{n}\left(r_{1}\right) . \\
& \text { do } n=-K-2, \cdots,-1 \\
& \text { do } l=2, \cdots, M-1
\end{aligned}
$$

$$
\begin{aligned}
& C_{n}\left(r_{l}\right)=\left(\frac{r_{l-1}}{r_{l}}\right)^{-n-2} C_{n}\left(r_{l-1}\right)+2 r_{l}^{n+2} C_{l-1, l}^{n}, \\
& B_{n}\left(r_{l}\right)=\left(\frac{r_{l-1}}{r_{l}}\right)^{-n} B_{n}\left(r_{l-1}\right)+2 r_{l}^{n} B_{l-1, l}^{n}, \\
& g_{n}\left(r_{l}\right)=C_{n}\left(r_{l}\right)-B_{n}\left(r_{l}\right) . \\
& \text { enddo }
\end{aligned}
$$

Step 6. Finally, compute

$$
\begin{aligned}
& G f\left(z=r_{l} e^{2 \pi i k / N}\right)=\sum_{n=-K-2}^{K-2} g_{n}\left(r_{l}\right) e^{2 \pi i k / N} ; \\
& l \in[1, M-1], k \in[1, N] .
\end{aligned}
$$

Algorithm 4.2 (The fast algorithm for solving the problem (1.1)):

Input: $M, N, \quad \gamma_{0}\left(e^{2 \pi i k / N}\right), \quad \gamma_{1}\left(e^{2 \pi i k / N}\right)$ and

$f\left(r_{l} e^{2 \pi i k / N}\right), \quad l \in[1, M-1], k \in[1, N]$.

Output: $w\left(r_{l} e^{2 \pi i k / N}\right), l \in[1, M-1], k \in[1, N]$.

Step 1. Set $K=N / 8, \quad r_{0}=0$ and $r_{M}=1$.

Step 2. Compute $G f\left(z=r_{l} e^{2 \pi i k / N}\right), \quad l \in[1, M-1]$, $k \in[1, N]$ using algorithm 4.1.

Step 3. Compute the Fourier coefficients $a_{n} \forall n \in[-K, K]$ from known values of $\gamma_{0}\left(e^{2 \pi i k / N}\right)$, $k=1,2, \cdots, N$ using the FFT.

Step 4. Compute the Fourier coefficients $b_{n} \forall n \in[-K-1, K-1]$ from known values of $\gamma_{1}\left(e^{2 \pi i k / N}\right)$, $k=1,2, \cdots, N$ using the FFT.

Step 5. Compute $u\left(r_{l} e^{2 \pi i k / N}\right), l \in[1, M-1], k \in[1, N]$ using the relation (2.1).

Step 6. Compute $v\left(r_{l} e^{2 \pi i k / N}\right), l \in[1, M-1], k \in[1, N]$ using the relation (2.3).

Step 7. Compute $w\left(r_{l} e^{2 \pi i k / N}\right), l \in[1, M-1], k \in[1, N]$.

$$
\begin{aligned}
& w\left(r_{l} e^{2 \pi i k / N}\right)=c r_{l} e^{2 \pi i k / N}+u\left(r_{l} e^{2 \pi i k / N}\right) \\
& +v\left(r_{l} e^{2 \pi i k / N}\right)+G f\left(r_{l} e^{2 \pi i k / N}\right)
\end{aligned}
$$

\section{Numerical Results}

In this section we solve a boundary value problem for the inhomogeneous Bitsadze equation in the unit disk by using the algorithms that presented in section 4 .

Example

Consider the problem

$$
w_{\overline{\mathrm{z}}}=2 z \text { in } B, w=\bar{z}, z w_{\overline{\mathrm{Z}}}=2, \forall z \in \partial B, \quad w_{\overline{\mathrm{z}}}(0)=0 \text {, }
$$

The exact solution for this problem is given by

$$
w(z)=\bar{z}^{2} z, \forall z \in \partial B
$$

By using algorithm 4.2 we have the max error as illustrate in the following Table 1. 
Table 1. Maximum relative error.

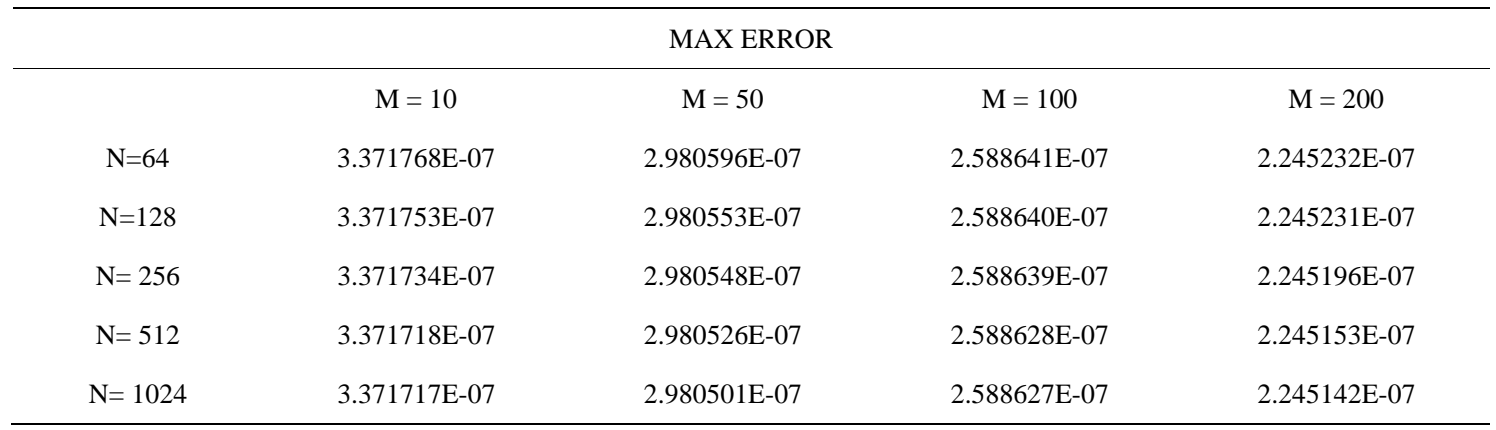

\section{Conclusions}

We presented a fast algorithm to solve the Bitsadze equation in the unit disk under special boundary conditions in the complex plane, by constructed the fast algorithm to evaluate the singular integral transform (1.4). The method divides the interior of the unit disk $B=$ $\{z:|z|<1\}$ into a collection of annular regions. The integrals and the function $f(z)$ are expanded in terms of Fourier series with radius dependent Fourier coefficients. The good performance of the algorithm is due to the use of scaling one-dimensional integral in the radial direction to produce the value of the singular integral over the entire domain. Specifically, scaling factors are used to define exact recursive relations which evaluate the radius dependent Fourier coefficients of the singular integral (1.4). The inverse Fourier transform are applied on each circle to obtain the value of the singular integrals on all circles.

\section{References}

[1] H. Begehr, "Boundary Value Problems in Complex Analysis I,” Boles' de la Association's Mathematical Venezuelan, Vol. 12, No. 1, 2005, pp. 65-85.

[2] H. Begehr, "Boundary Value Problems in Complex Analysis II,” Boles' de la Association' Mathematical Venezuelan, Vol. 12, No. 2, 2005, pp. 217-250.

[3] P. Daripa, "A Fast Algorithm to Solve Nonhomogeneous Cauchy-Riemann Equations in the Complex Plane," SIAM Journal on Scientific and Statistical Computing, Vol. 13, No. 6, 1992, pp. 1418-1432. doi:10.1137/0913080

[4] P. Daripa and D. Mashat, "An Efficient and Novel Nu- merical Method for Quasiconformal Mappings of Doubly Connected Domains," Numerical Algorithms, Vol. 18, No. 2, 1998, pp. 159-175. doi:10.1023/A:1019169431757

[5] P. Daripa and D. Mashat, "Singular Integral Transforms and Fast Numerical Algorithms," Numerical Algorithms, Vol. 18, No. 2, 1998, pp. 133-157. doi:10.1023/A: 1019117414918

[6] P. Daripa and L. Borges, “A Fast Parallel Algorithm for the Poisson Equation on a Disk," Journal of Computational Physics, Vol. 169, No. 1, 2001, pp. 151-192. doi: 10.1006/jcph.2001.6720

[7] P. Daripa and L. Borges, "A Parallel Version of a Fast Algorithm for Singular Integral Transforms,” Numerical Algorithms, Vol. 23, No. 1, 2000, pp. 71-96. doi:10.1023/ A:1019143832124

[8] P. Daripa, "A Fast Algorithm to Solve the Beltrami Equation with Applications to Quasiconformal Mappings," Journal of Computational Physics, Vol. 106, No. 2, 1993, pp. 355-365.

[9] P. Daripa, “On a Numerical Method for Quasiconformal Grid Generation,” Journal of Computational Physics, Vol. 96, No. 2, 1991, pp. 229-236. doi:10.1016/0021-9991(91) 90274-O

[10] A. H. Babayan, “A Boundary Value Problem for Bitsadze Equation in the Unit Disc," Journal of Contemporary Mathematica Analysis, Vol. 42, No. 4, 2007, pp. 177-183. doi:10.3103/S1068362307040012

[11] A. V. Bitsadze, "Boundary Value Problems of Second Order Elliptic Equations," North-Holland Publishing Company, Amsterdam, 1968.

[12] C. Miranda, "Partial Differential of Elliptic Type," Springer, New York, 1970.

[13] M. Schechter, L. Bers and F. John, "Partial Differential Equations,” Interscience, New York, 1964. 